\title{
Smart Irrigation System Using Internet of Things
}

\author{
Babanna Kumbar, Basavaraj Galagi, Bheemashankar and Naveen Honnalli
}

\begin{abstract}
The paper describes the smart irrigation system using the concept of IoT. The project uses a Wi-Fi module (ESP8266-12) which connects the system to internet. This module controls a motor and two solenoid valves for supplying water to the field on the information obtained from a water level indicator and two soil moisture sensors. This whole system is monitored and controlled by MQTT server (My MQTT android App) through internet. The paper also depicts the concept of Internet of Things (IoT).
\end{abstract}

Keyword--- IoT-Internet of Things, API-Application Programming Interface, IP-Internet Protocol, MQTT-Message Queue Telemetry Transport, IDE-Integrated Development Environment

\section{INTRODUCTION}

I NDIA is the country of village and agriculture plays an important role for development of country. In our country, agriculture depends on the monsoons which has insufficient source of water. So the irrigation is used in agriculture field. In Irrigation system, depending upon the soil type, water is provided to plant. In agriculture, two things are very important, first to get information of about the fertility of soil and second to measure moisture content in soil. Nowadays, for irrigation, different techniques are available which are used to reduce the dependency of rain. And mostly this technique is driven by electrical power and on/off scheduling. In this technique, water level indicator placed in water reservoir and soil moisture sensors are placed root zone of plant and near the module and gateway unit handles the sensor information and transmit data to the controller which in turns the control the flow of water through the valves.

Fig 1 shows the basic architecture of IoT. The Internet of Things (IoT) is the network of physical objects devices, vehicles, buildings and other items embedded with electronics, software, sensors, and network connectivity that enables these objects to collect and exchange data. The IoT allows objects to be sensed and controlled remotely across existing network infrastructure, creating opportunities for more direct integration of the physical world into computer based systems, and resulting in improved efficiency, accuracy and economic benefit, when IoT is augmented with sensors and actuators, the technology becomes an instance of the more general class of cyber physical systems, which also encompasses technologies such as smart grids, smart homes, intelligent transportation and smart citiesSo, Internet of Things or IoT is an architecture that comprises specialized hardware boards, Software systems, web APIs, protocols which together creates a seamless environment which allows smart embedded devices to be connected to internet such that sensory data can be accessed and control system can be triggered over internet.

Also devices could be connected to internet using various means like Wi-Fi, Ethernet and so on. Furthermore devices may not needed to be connected to internet independently. Rather a cluster of devices could be created (for example a sensor network) and the base station or the cluster head could be connected to internet. This leads to more abstract architecture for communication protocols which ranges from high level to low level.

Most interestingly, these devices must be uniquely discovered. For unique discovery of the devices in a Network, they need to have unique IP address. IoT devices essentially have IPv6 addressing scheme. All these devices have either fixed or Subnet masked IP addresses of type v6. Unique IP addresses makes IoT devices discoverable in the internet as independent node. This is the most important concept to have in mind to understand IoT.

This paper uses concept of IoT for monitoring and controlling the system using a public server called MQTT server. It uses an android app called My MQTT. In this app, one has to subscribe a topic and publish a message of specific function. The server will call-back to perform the function.

MQTT stands for Message Queue Telemetry Transport. It is publish/subscribe, extremely simple and lightweight messaging protocol, designed for constrained devices and lowbandwidth, high-latency or unreliable networks. These principles also turn out to make the protocol ideal of the emerging "machine-to-machine" (M2M) or "Internet of Things" world of connected devices, and for mobile applications where bandwidth and battery power are at a premium. 


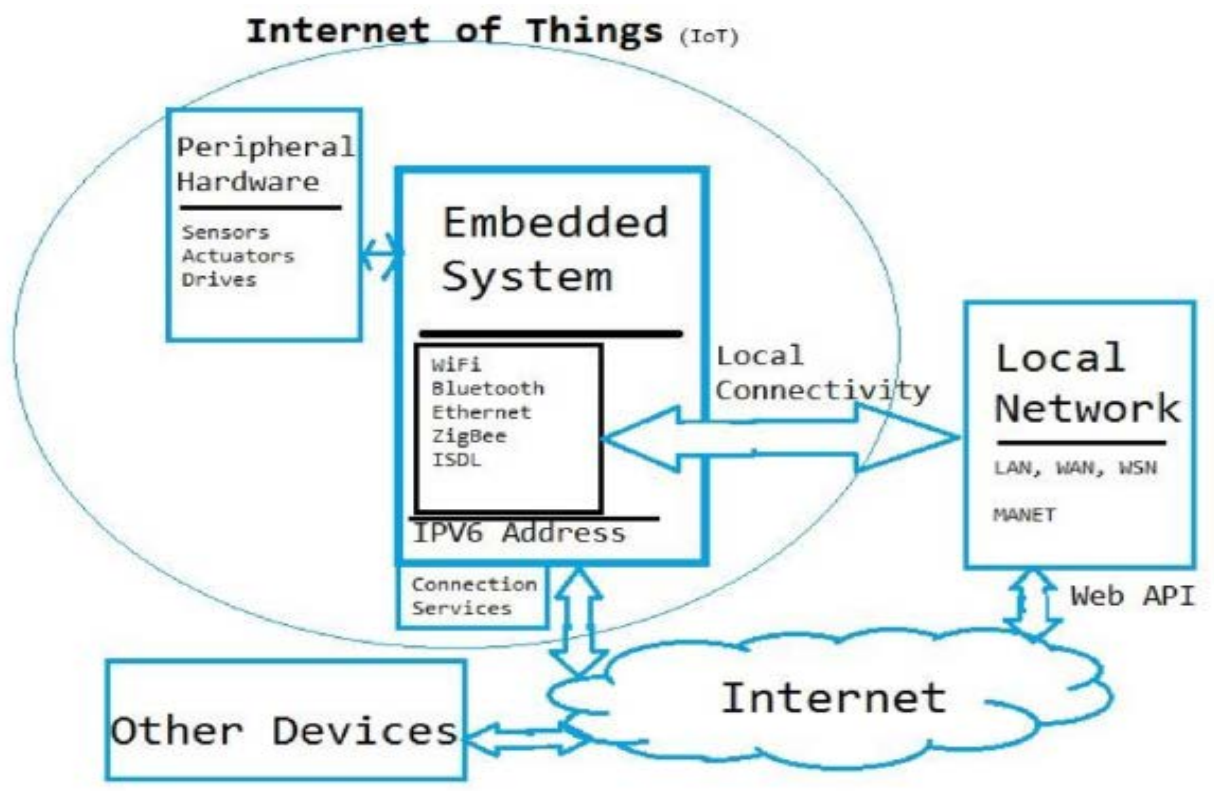

Figure 1: Basic Architecture of IoT

\section{EXPERIMENTAL SETUP}

\subsection{Software Development}

Initial Setups in Arduino IDE Software

Step1: Install the Arduino 1.6.7 IDE.

Step2: Go to File>>Preferences>>Additional Boards Manager URLs:

http://arduino.esp8266.com/stable/package_esp8266com_ index.json $>>\mathrm{Ok}$

Step 3: Go to Tools $>>$ Boards $>>$ Boards Manager $>>$ Download the "esp8266 by ESP8266 Community version 2.2.0"

Step4: Go to Tools>>Boards>>Generic ESP8266 Module

Step5: Go to Tools >>Upload Speed $>>11520>>$

Port>>choose preferred COM ports.

\subsection{Hardware Development}

\section{Components Required}

Table 1: Components Required

\begin{tabular}{|l|l|}
\hline Components & Quantity \\
\hline Explore ESP8266 Wi-Fi Module & 1 \\
\hline Relay Switch & 3 \\
\hline Transistor (BC-547) & 3 \\
\hline Diode (IN4007) & 3 \\
\hline LED (Power Indication) & 1 \\
\hline
\end{tabular}

\section{Circuit Connections}

Step1: Make the connection in bread-board as shown in fig 2 and verify results.

Step2: Once results are confirmed, make a PCB layout using software Express PCB as shown below in figure 2.2.

Step3: Print the PCB layout on a copper plate.
Step4: Itching: Immerse the printed PCB copper plate in a copper sulphate solution until all copper oxidizes except PCB traces. Then, wash with petrol to remove printed carbon.

Step5: Place the components and carefully solder them. Front and rear views are shown in fig4 and fig5.

Step6: Make the complete connections, i.e. connect the water level indicator, soil moisture sensors, and submersible motor pump and solenoid valves.

Step7: Connect the power supplies to output ends of relay switches as mentioned in the circuit diagram. $9 \mathrm{v}$ to motor pump, $18 \mathrm{v}$ to both valves. Connect the power supplies to PCB board $5 \mathrm{v}, 3.3 \mathrm{v}$ and ground from Arduino board.

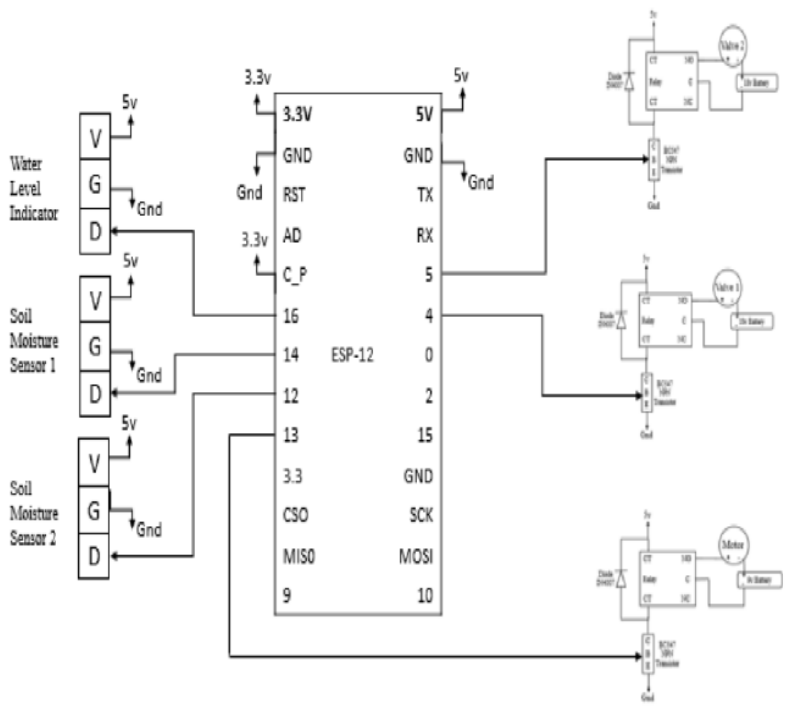

Figure 2: Circuit Diagram 


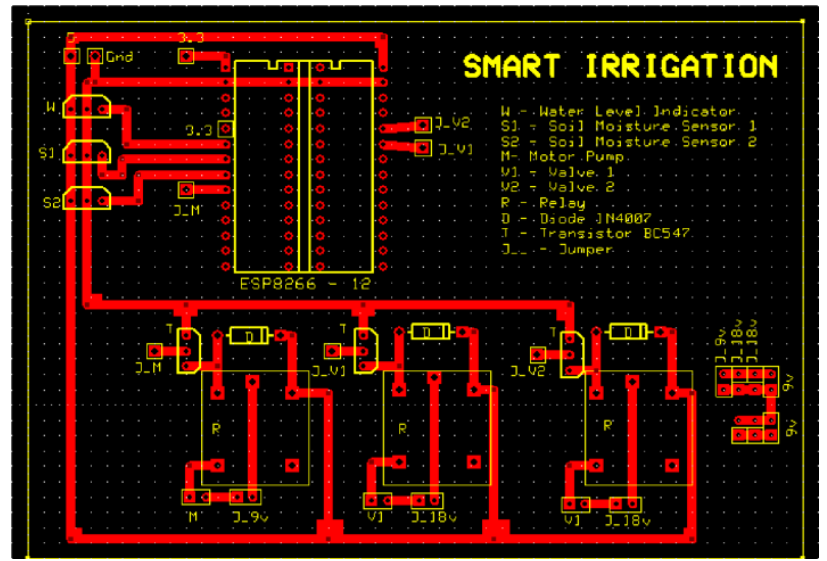

Figure 3: PCB Layout

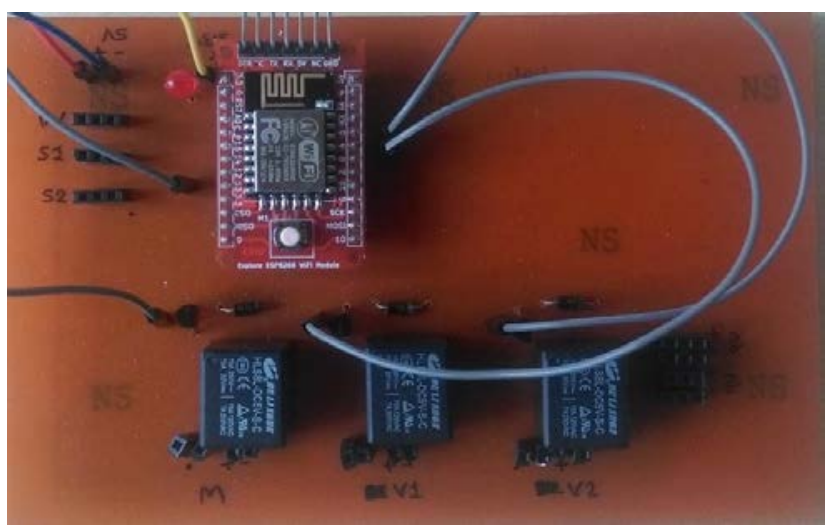

Figure 4: Front View of PCB Layout

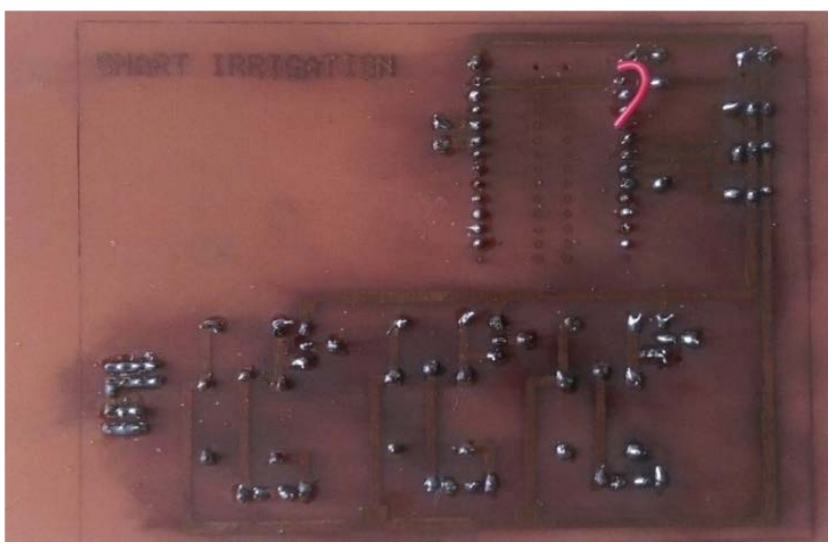

Figure 5: Rear View of PCB Layout

\section{A. Physical Connection}

Physical connections include the placing the sensors and actuators in small model of agriculture field and includes proper connections. Detail is given below.

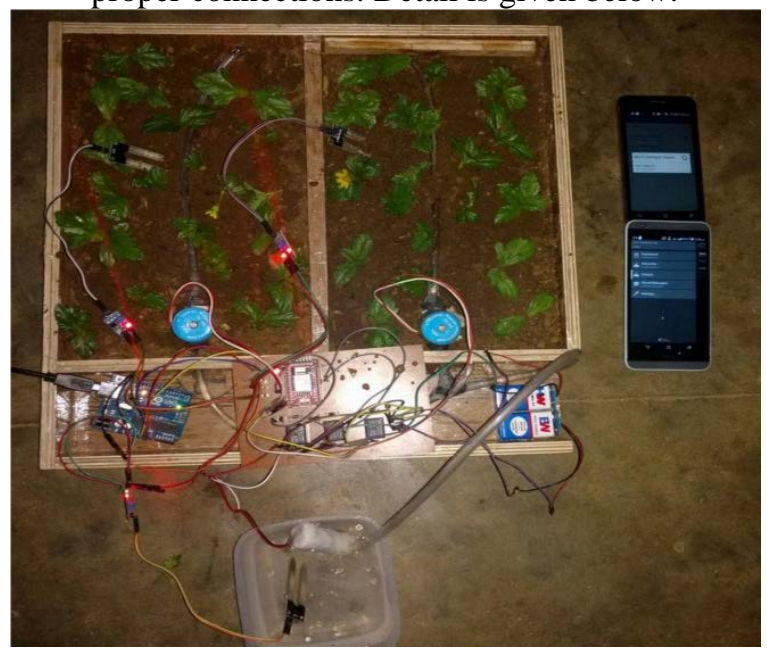

Figure 6: Physical Connection

1. The field includes two Regions: 1, 2 and a water reservoir.

2. Submerse the submersible motor pump in the reservoir.

3. Place the valve 1 in Region 1.

4. Place the valve 2 in Region 2.

5. Make proper pipeline connections from motor pump to the valves.

6. Extend the pipeline connection to respective fields to supply water.

7. Make some arrangements to supply the water like making the holes to pipes.

8. Place the water level indicator in the water reservoir.

9. Place the soil moisture sensor 1 in Region 1 near the roots of the plants.

10. Place the soil moisture sensor 2 in Region 2 near the roots of the plants.

11. Give all required supply voltages.

Physical connection and implementation is shown in fig6.

\section{RESULTS AND VERIFICATION}

\subsection{How Module Works?}

1. Wi-Fi module has to connect the internet by an internet service provider like mobile hotspot, Wi-Fi router.

2. Firstly module checks status of water level indicator, if water is present then it proceeds otherwise it terminates.

a) If water is present, then it checks status of soil moisture sensor 1 ,

b) If Region 1 is wet, motor will be off.

c) If Region 1 is dry, valve 1 will open and motor will be on for 10 seconds.

d) If Region 1 is humid, valve 1 will open and motor will be on for 5 seconds.

e) During this, valve 2 will remain closed.

3. Once again module checks status of water level indicator, if water is present then it proceeds otherwise it terminates. 
a) If water is present, then it checks status of soil moisture sensor 2 ,

b) If Region 2 is wet, motor will be off.

c) If Region 2 is dry, valve 2 will open and motor will be on for 10 seconds.

d) If Region 2 is humid, valve 2 will open and motor will be on for 5 seconds.

e) During this, valve 1 will remain closed.

1. System is usually OFF state.

2. It is possible to get STATUS of the field.

3. It is possible to make System ON whenever. Once System is ON, it will check the status and supply the water one time only. Then again System is OFF.

4. It is possible to make System OFF. But usually System is always will OFF condition.

\subsection{Controlling the Module Using My MQTT Android APP}

My MQTT is a simple Message Queue Telemetry Transport (MQTT) client for Android. The service is being provided by Mosquit to of eclipse a service provider.

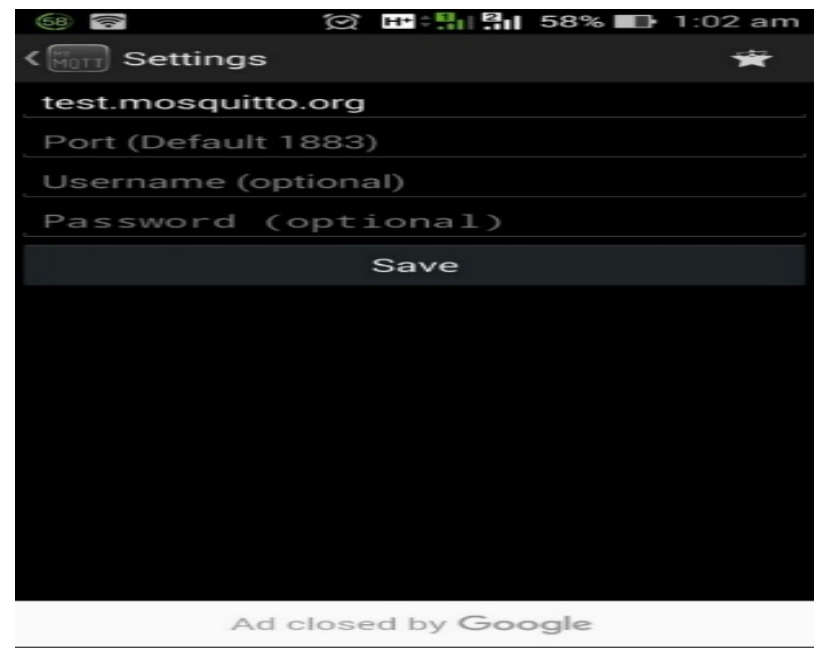

Figure 7: Initial Setup

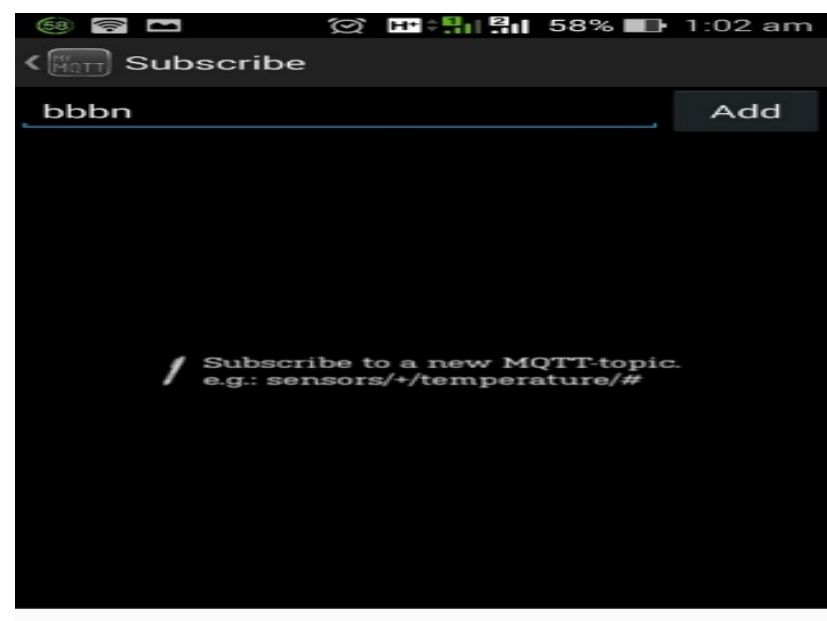

Ad closed by Google

Figure 9: Subscribing a Topic
Features: Connect to MQTT v3.1 Broker (optional with username and password) Subscribe to various topics. Publish messages to a topic Save messages Filter received Messages Step1: Initial setups in app is to make. Go to Settings $>>$ Broker URL>>”test.mosquitto.org” (Fig 7). Then client will connect to Broker URL (Fig 8).

Step2: Subscribe to a topic called bbbn. Go to Subscribe>>” bbbn">>Add (Fig 9). Once subscription is successful, then messages will be received in Dashboard.

Step3: Publish a message. Go to Publish>>Topic>>"bbbn" $>>$ "-message-“>>Publish (Fig 10). Then message will be published in Dashboard and broker server. "-messages“may be STATUS, SYSTEM_ON, SYSTEM_OFF>

Depending upon messages published, different functions will be executed and client will publish in Dashboard what it do? What it will be does? What it done?

The following figures shows screenshots of Dashboards depending upon Publish.
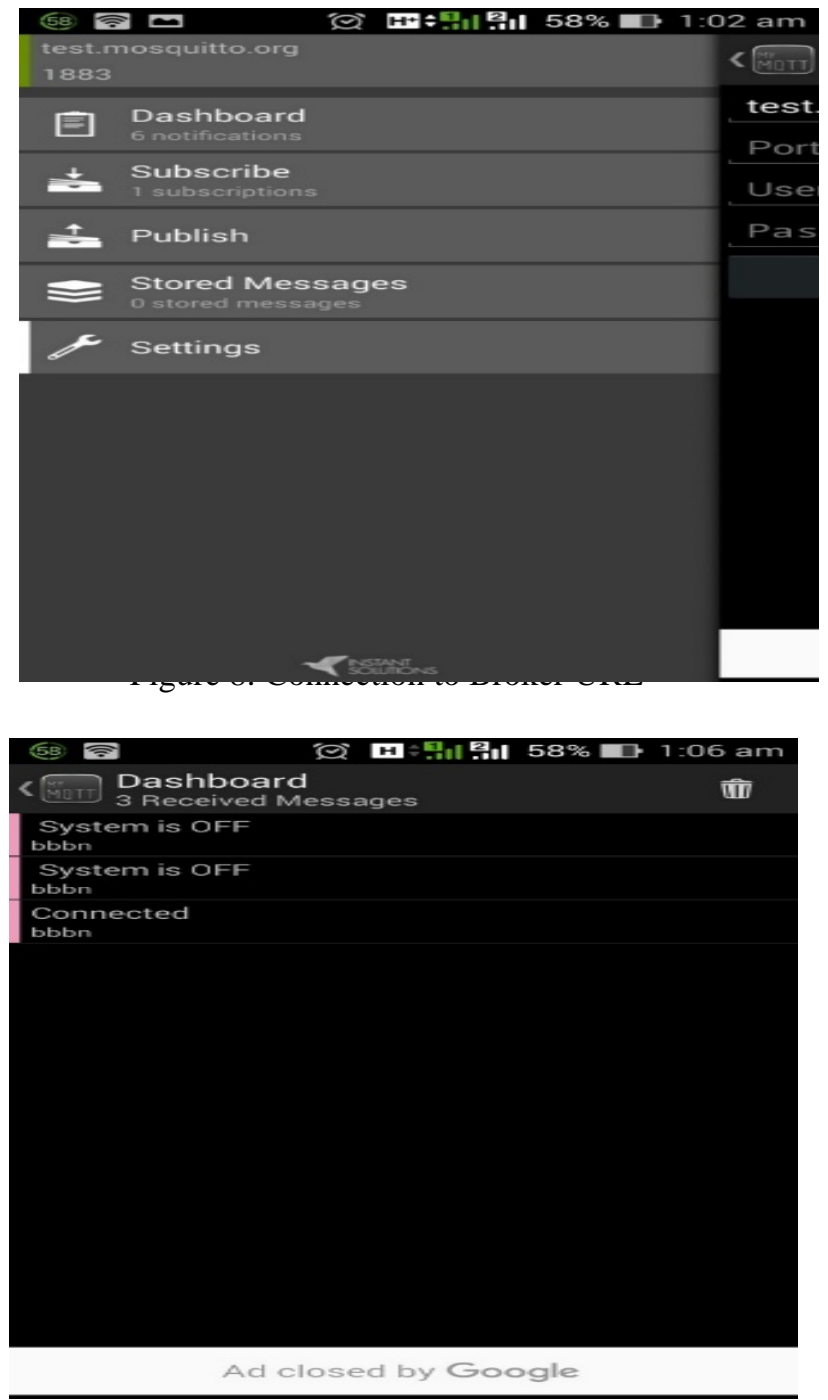

Figure 10: Messages in Dashboard 


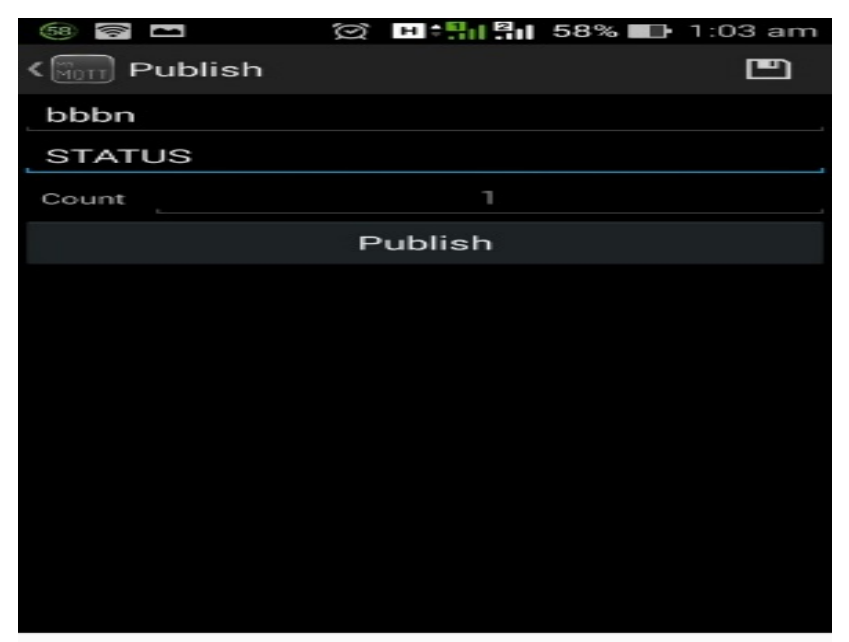

Ad closed by Google

Figure 11: Publish of Message(STATUS)

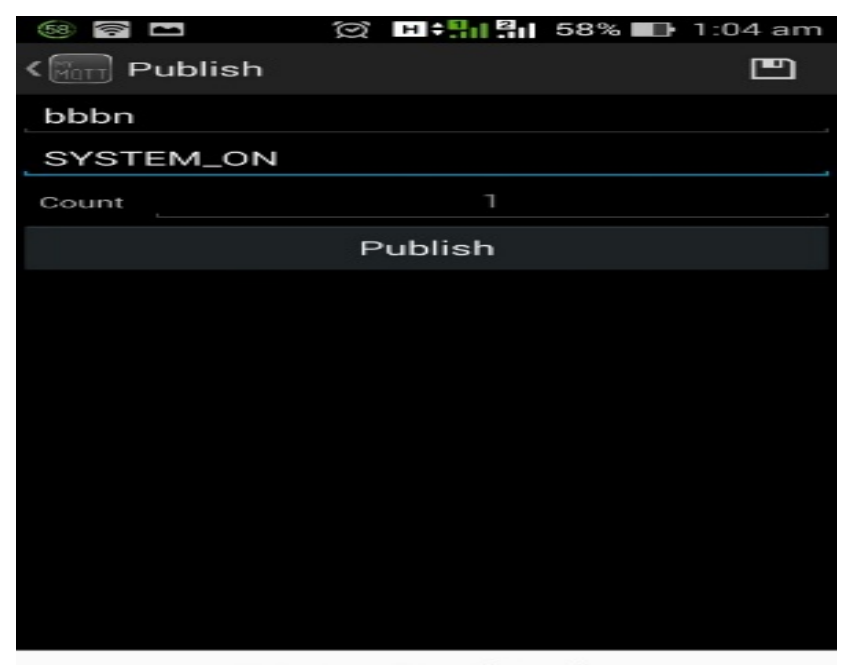

Ad closed by Google

Figure 13: Publish of Message(SYSTEM_ON)

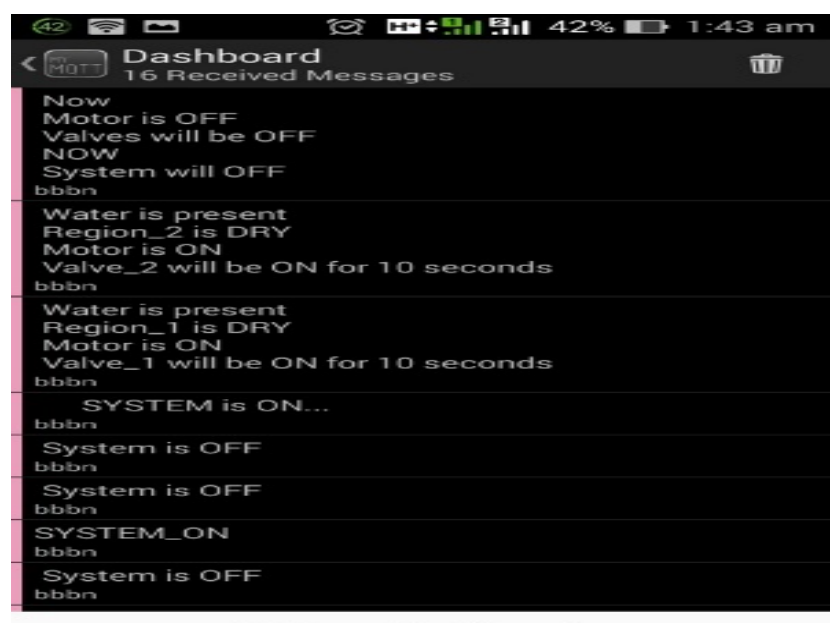

Ad closed by Google

Figure 15: Messages on Dashboard(SYSTEM_ON)

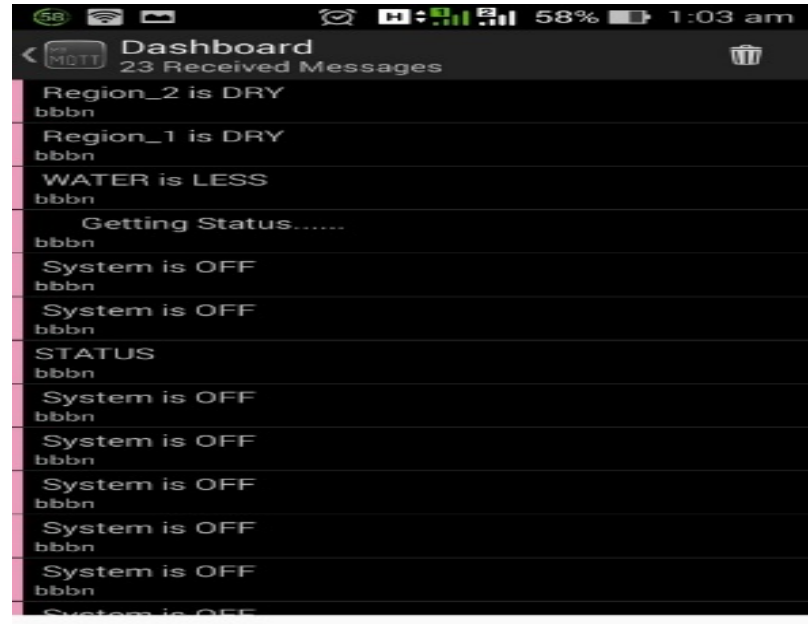

Ad closed by Google

Figure 12: Messages on Dashboard due to STATUS

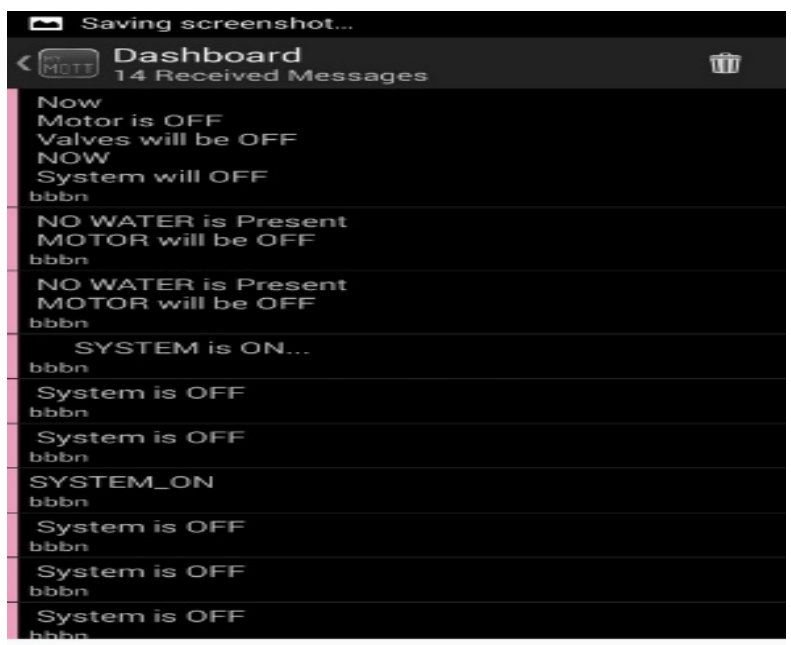

Ad closed by Google

Figure 14: Messages on Dashboard(SYSTEM_ON)

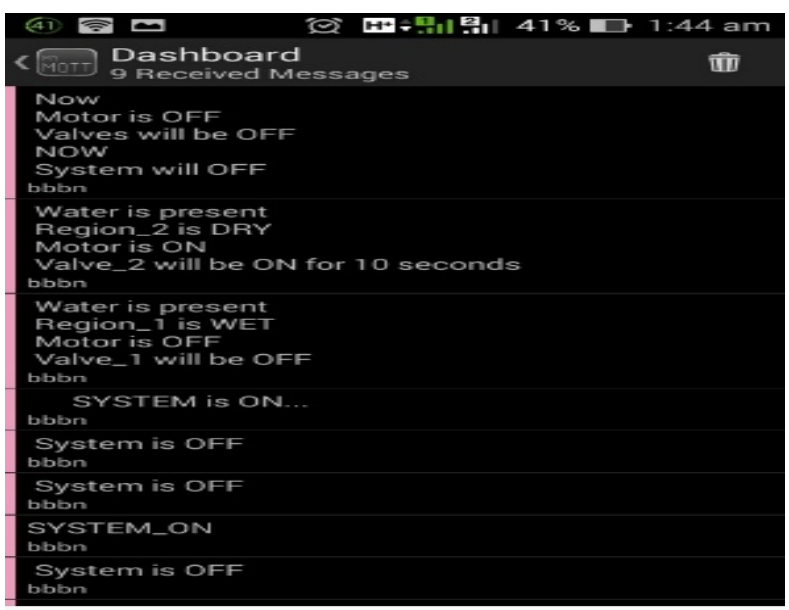

Ad closed by Google

Figure 16: Messages on Dashboard(SYSTEM_ON) 


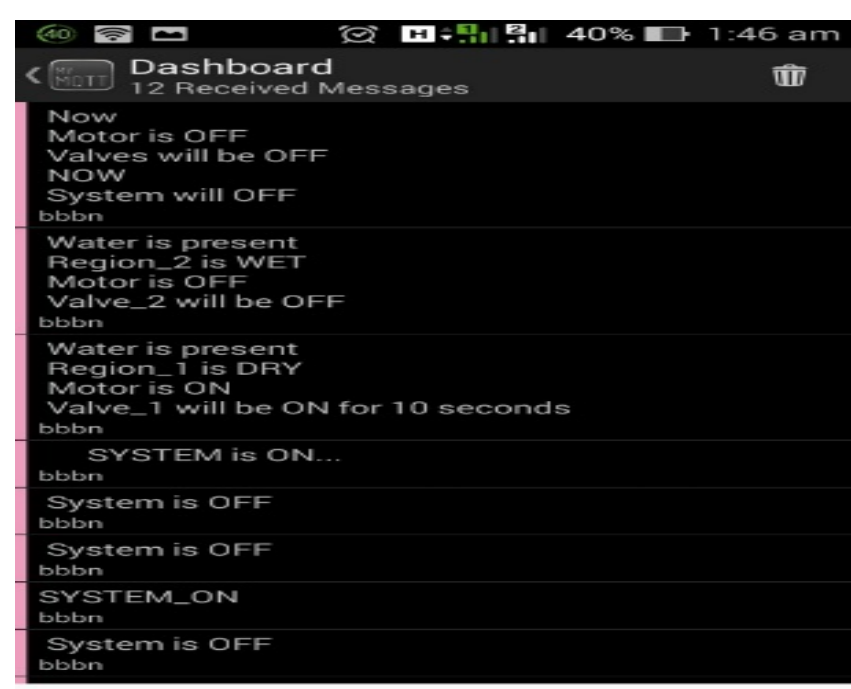

Ad closed by Google

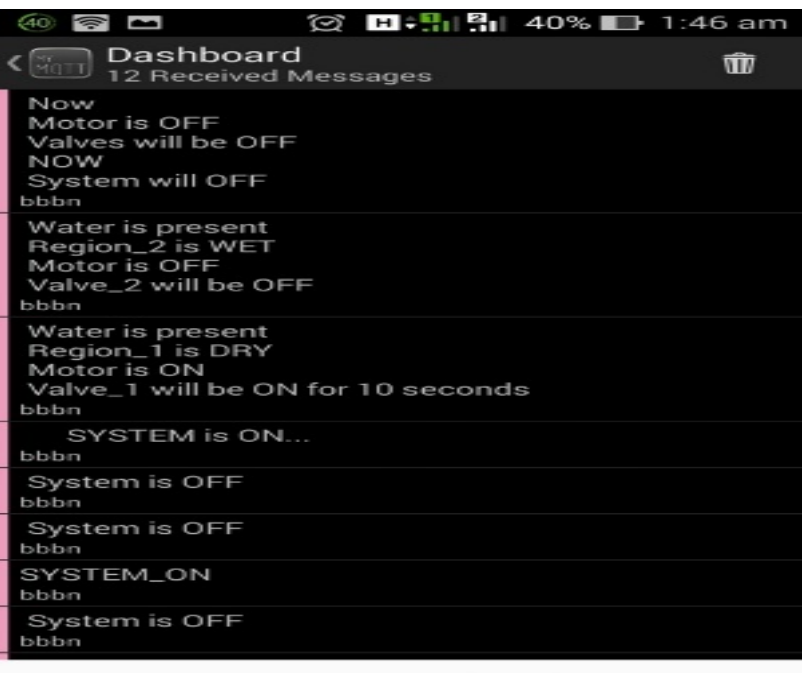

Ad closed by Google

Figure 18: Messages on Dashboard(SYSTEM_ON)

\section{CONCLUSION}

The agriculture field is being monitored and controlled by My MQTT android app at user end. The ESP8266 is the device at field end which receives the messages from broker network and manipulates it and will perform the function mentioned in message. After it will send the messages to broker network and in turn it will be published to the Client (user end). The ESP8266 is the best device for IoT projects. Since it is small, compact, lightweight, easily programmable, and easily installable and have enough GPIO pins to use them.

\section{REFERENCES}

[1] M.D. Rane, P.G. Scholar-VLSI and S. BDCE, "REVIEW PAPER BASED ON AUTOMATIC IRRIGATION SYSTEM BASED ON RF MODULE”, Vol.1, No.9, 2014.

[2] Sensor Based Automated Irrigation System With Iot: A Technical Review by Karan Kanasura, Vishal Zaveri, Babu Madhav Institute of Technology, Uka Tasadia University, Bardoli, Gujarat, India.

[3] Intelligent Automatic Irrigation System, by Dr. Sarika Tale, Sowmya P, Associate Professor department of Digital Electronics and Communication Systems, Visvesvaraya Institute of Advanced Technology, Muddenahalli, Chikkaballapur, India.

[4] R. Thakur and D. Singh, "Microcontroller based automatic sprinkler irrigation system”, International journal of modern engineering research (IJMER), Vol.5, No.4, 2015.

[5] S.S. Belsare, "Design and Implementation of Automated Irrigation System ZIGBEE and GSM", International Journal of scientific research and management(IJSRM), Vol.3, No.6, Pp.3027-3030, 2015. 